\title{
A 2D Method For Acquiring The Radiation Pattern Of Two-Identical Vivaldi Antennas By Using A Stepped-Frequency Continue Wave (SFCW) Radar And A Rotation Stage
}

\author{
J. L. Solano, V. Ortega, R. S. Silla, W. Schröder, L. Schüssele \\ IUAS Institute of Unmanned Aerial Systems, Offenburg University of Applied Sciences, Germany \\ \{jorge.solano, valentin.ortega\} @ hs-offenburg.de
}

\begin{abstract}
A simple measuring method for acquiring the radiation pattern of an ultra-wide band Vivaldi antenna is presented. The measuring is performed by combining two identical Vivaldi antennas and some of the intrinsic properties of a stepped-frequency continue wave radar (SFCW radar) in the range from $1.0 \mathrm{GHz}$ to $6.0 \mathrm{GHz}$. A stepper-motor provided the azimuthal rotation for one of the antennas from $0^{\circ}$ to $360^{\circ}$. The tests have been performed within the conventional environment (laboratory / office) without using an anechoic chamber or absorbing materials. Special measuring devices have not been used either. This method has been tested with different pairs of Vivaldi antennas and it can be also used for different ones (with little or no change in the system), as long as their operational bandwidth is within the frequency range of the SFCW radar.

Keywords - SFCW Radar, Antenna Gain Characterization, Azimuthal Radiation Pattern
\end{abstract}

\section{INTRODUCTION}

For certain applications and under specific conditions, the information about certain parameters of an antenna are required, such as the radiation pattern at certain frequency or frequency range. Depending on the approach and the method applied, it might result in a complex and expensive task, as high-end equipment is required[1] and in occasions an anechoic chamber too[2][3]. In cases where an anechoic chamber is not available, the measurements can take place in open spaces[4]. However this is not always an appropriate option as it implies weather dependency and/or issues with the equipment availability and mobility.

Antennas can be characterized by using different methods: absolute gain, two-identical antenna, direct comparisson, etc.[5]. The applied method will be normally chosen according to the requirements of the system and equipment at disposal. In any case, some device must be connected to the antennas in order to generate the excitation signal on a defined frequency range and at least a second antenna must serve as receiver.

The Vivaldi antenna, first proposed by Gibson in 1979 [6], is commonly used for ultra-wide band radar applications due to its large bandwidth, low-cost, compactness, etc[7]. Therefore, in order to characterize these antennas it is necessary to use equipment, such as excitation sources and receivers, that meet these large bandwidths requirements.
In the present paper we have used a combination of the two-identical antenna method and a SFCW radar. The description of antenna gain has been obtained with a modified version of the Friss-equation[8, p. 165]:

$$
G_{A U T}=\frac{1}{2}\left[20 \log _{10}\left(\frac{4 \pi R}{\lambda}\right)+10 \log _{10}\left(\frac{P_{r}}{P_{t}}\right)\right],
$$

where $R$ is the distance between the two antennas under test (AUT), $\lambda$ is the operating wavelength of the signal, and $P_{r} / P_{t}$ is the power ratio of the transmitted and the received signals.

As excitation source we have used a SFCW radar that can be effortless configured to emit signals in the range from 1.0 $\mathrm{GHz}$ to $6.0 \mathrm{GHz}$ in steps of fractions of $\mathrm{MHz}$. Additionally a rotating unit attached to a stepper-motor has been used for scanning the azimuth of the AUT.

The objective of this experiment was to determine the similarity between the simulated and the real radiation pattern of the Vivaldis. The satisfying results of this method could imply a possible reduction of time, effort, and costly and complex facilities that are required for these type of antenna measurements.

\section{MATERIALS, EQUiPMENT AND METHODOLOGY}

The experimental setup consists of two identical Vivaldi antennas, a SFCW radar operating in the frequency range from 1.0 $\mathrm{GHz}$ to $6.0 \mathrm{GHz}$, and a rotary stage attached to a stepper motor.

The setup has been driven by using a script based on tools from GNU/Octave, which is a high-level programming

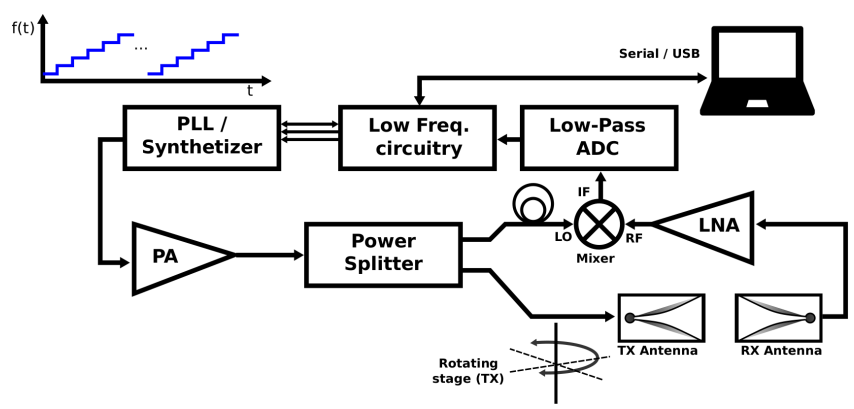

Fig. 1. Basic diagram of the setup for the measurement 
language for numerical computations[9] that was running on a PC. These tools, besides driving the measuring setup, also allow the evaluation of the data obtained in each test in a effortless fashion.

Figure 1 shows a diagram with a general representation of the measuring setup used for the characterization of the Vivaldi antennas.

\section{A. The Vivaldi antennas}

The antennas used for the measurements have been designed, modified and simulated by using Antenna Magus ${ }^{\circledR}$ and CST Microwave Studio ${ }^{\circledR}$. On the first step, we have generated a Vivaldi antenna from Antenna Magus. This antenna, with a lower frequency at $3.0 \mathrm{GHz}$, has been exported and subsequently modified and repeatedly simulated on CST Microwave Studio until the desired characteristics of size, radiation patterns, reflections, and impedance have been obtained in the range from $1.0 \mathrm{GHz}$ to $6.0 \mathrm{GHz}$.

The resulting Vivaldi antenna has an overall size of $160 \mathrm{~mm}$ by $135 \mathrm{~mm}$ and it has been manufactured with copper on top of a FR-4 substrate, having a thickness of $1.55 \mathrm{~mm}$ and an $\varepsilon_{r}=4.3$. The link between the antennas and the SFCW radar is accomplished by placing coaxial connectors of the type SMA. Figure 2 shows the main dimension values of the Vivaldi antenna.

\section{B. Stepped frequency continuous wave radar}

As excitation and receiving device for the characterization of the antennas, we have use a SFCW radar. The SFCW Radar has been designed to operate in the same frequency range as the Vivaldi antennas described previously in II-A. As shown in figure 1, the SFCW radar is a simple combination of two amplifiers, one frequency mixer, a power splitter, a frequency synthesizer, and digital low-frequency circuitry. This setup is driven by a personal computer through the USB interface. The measurements have been performed by using GNU/Octave scripts. This has allowed a rapid integration of the radar with the rotation stage and the evaluation process.

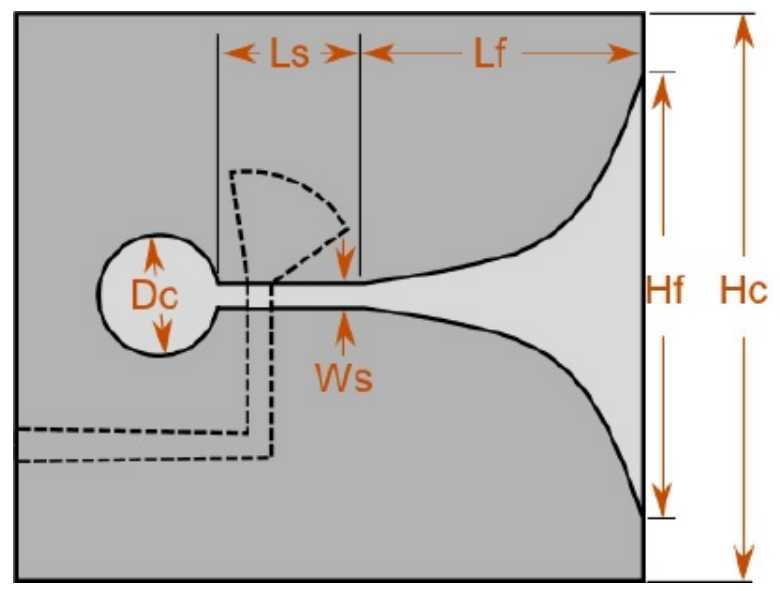

Fig. 2. Physical characteristics of the modified antenna (image taken from Antenna Magus). Flare length (Lf) $=142 \mathrm{~mm}$, flare height $(\mathrm{Hf})=124 \mathrm{~mm}$, height of conductor $(\mathrm{Hc})=134 \mathrm{~mm}$
The SFCW radar can be configured to display frequencies in the range from $1.0 \mathrm{GHz}$ to $6.0 \mathrm{GHz}$ with frequency steps $(\Delta F)$ of only fractions of $\mathrm{MHz}$. For the experiments reported in the present paper, we have used values of $\Delta F=1.0 \mathrm{MHz}$, 2.0 MHz, 5.0 MHz, $10.0 \mathrm{MHz}$, and 20.0 MHz. The amount of data points obtained for each measurement is inversely proportional to the size of the frequency step, and they can be calculated with the following equation:

$$
\text { DataPoints }=\frac{\text { StopFreq }- \text { StartFreq }}{\Delta F}
$$

The amount of data points (DataPoints in 2) must be chosen depending on the electrical path difference observed by the frequency mixer on the SFCW Radar. The larger the difference, the higher frequency will appear at the $I F$ signal and hence a higher sampling is required and therefore smaller values of $\Delta F$ will be necessary.

Prior to the measurements, a calibration procedure is performed in order to verify the output signal of the radar. The calibration is accomplished by transferring a series of pre-calculated registers to the synthesizer. By substituting the antennas with a coaxial cable (and a $30 \mathrm{~dB}$ attenuator) of similar electrical path length as the separation of the antennas it is possible to analyze the signal that we are expecting.

Figure 3 shows the output signal ( $I F$ or beat signal after the frequency mixer) generated by the radar with a cable loop and its spectrum (where the electrical path difference can be demonstrated). The beat frequency can be calculated by using the following formula:

$$
f_{b}=\frac{N \Delta F}{T_{b}} \frac{2 R}{v}
$$
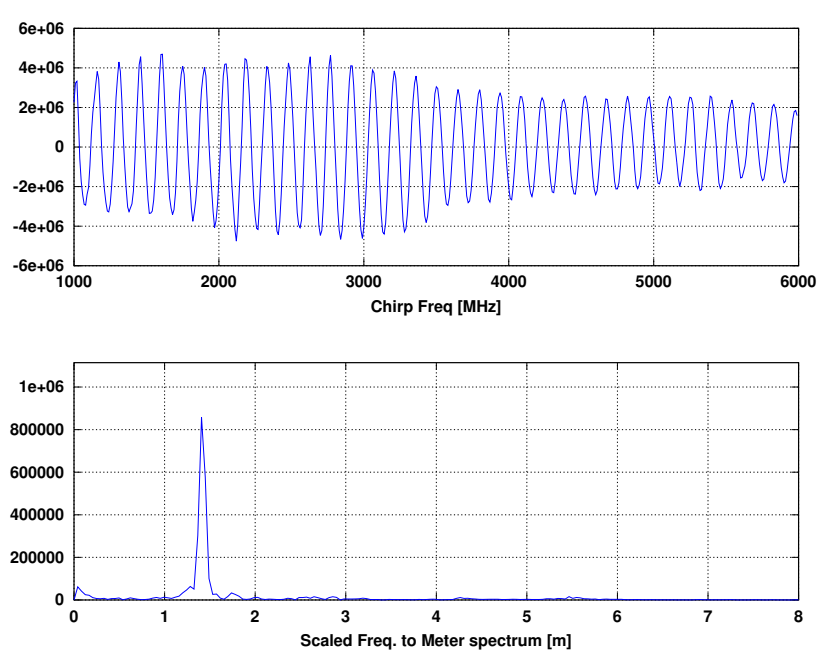

Fig. 3. Beat signal generated by sweeping from $1.0 \mathrm{GHz}$ to $6.0 \mathrm{GHz}$ in steps of $10.0 \mathrm{MHz}$ (500 data points) and its frequency-to-meters scaling Fourier Transformation obtained with the SFCW radar with a loop of coaxial cable. This kind of test has helped defining the distance between the Vivaldi antennas 
where $\Delta F$ is the frequency step, $N$ is the total amount of data points, $T_{b}$ is the total sweep time, $R$ represent the path difference and $v$ is the speed of propagation of the wave.

Once the calibration test has been successfully carried out, we have proceeded with the measurements with the actual Vivaldi antennas.

\section{Antenna measurements and data evaluation}

Both transmitting (TX) and receiving (RX) Vivaldis are placed in wood holder at a separation distance of approximately $0.75 \mathrm{~m}$. The TX antenna has been mounted on a rotation stage, which allows this antenna to cover the required azimuth angles.

Right after the calibration procedures, the SFCW radar starts sweeping the frequencies calculated at the first angle position (generally $0^{\circ}$ ). Simultaneously the radar is also sampling the signal from the receiving antenna. This will be repeated until all the programmed angles of the TX antenna have been covered. The sampled data from the radar is transferred to the PC application for evaluation. For convenience, this information is stored on a matrix with the form

$$
\operatorname{data}(F, \phi)=\left[\begin{array}{cccc}
\left(F_{1}, \phi_{0^{\circ}}\right) & \left(F_{1}, \phi_{5^{\circ}}\right) & \ldots & \left(F_{1}, \phi_{360^{\circ}}\right) \\
\left(F_{2}, \phi_{0^{\circ}}\right) & \left(F_{2}, \phi_{5^{\circ}}\right) & \ldots & \left(F_{2}, \phi_{360^{\circ}}\right) \\
\vdots & \vdots & \vdots & \vdots \\
\left(F_{N}, \phi_{0^{\circ}}\right) & \left(F_{N}, \phi_{5^{\circ}}\right) & \ldots & \left(F_{N}, \phi_{360^{\circ}}\right)
\end{array}\right]
$$

The amount of data stored in this matrix depends on the angle resolution and on the chosen size of $\Delta F$. For most of the tests carried out, an angle resolution of $5^{\circ}$ and a frequency step size of $\Delta F=10.0 \mathrm{MHz}$ have been used. These parameters generated a adequate amount of information for analyzing the radiation pattern of the Vivaldi antenna.

The evaluation of the sampled data makes use of the knowledge about the fixed distance between the TX and RX Vivaldi antennas. This aforementioned fixed distance can be easily detected on the spectrum as the main peak of the signal (beat frequency $f_{b}$ ). The sampled data from the SFCW radar also contains information about the surrounding objects where the measurement took place. Nevertheless, the signal from these objects can be easily filtered out and only the signal from the path difference will generally dominate.

From the remaining signal we have calculated the power distribution along the chirp generated for each angle. Subsequently we have been applied equation 1 (the modified Friss-equation) in order to determine the gain of the antenna at each position. With these results we have generated the polar diagram for the desired frequencies.

\section{EXPERIMENTAL RESULTS}

The different tests carried out by using the measurement method described in section II have shown results of radiation patterns that correspond to those obtained during the simulation phase on CST Microwave Studio. We have found certain discrepancies in certain frequency ranges that are related to the filtering of the data obtained from the SFCW radar and some random noise from the circuitry.

For comparison purposes, the same type of measurements have been carried out with additional absorber walls around the antennas setup. The results have shown non-relevant changes on the final results. This effect was to be expected, as the radar signal generated by the fix distance between the two antennas is the one dominating in our evaluation method.

In that sense, we would expect a signal with similar frequencies values (to the value of the signal generated by the fixed distance between our antennas) if, for instance, a metal plate or foil comes parallel and close to our antenna pair.

A complete azimuthal measurement takes only a few minutes (depending on how the angle and frequency resolution have been configured). This also include the evaluation procedures (which only take a few fractions of a second). Besides the measuring process, the data evaluation has been also accomplished by using the adequate set of tools from GNU/Octave.

Since we have used the two-identical antennas method (by directly applying the modified version of the Friss-Equation), it has not been necessary to perform additional compensation procedures in order to eliminate the effects of the AUT.

Figure 4 shows a comparative set of polar plots of the Vivaldi antenna. In this figure we have used frequency chirps in the range from $1.0 \mathrm{GHz}$ to $6.0 \mathrm{GHz}$ with a $\Delta F=10.0 \mathrm{MHz}$. This combination of parameters generate 500 points of data at the receiver side of the SFCW radar for each angular position.

The frequency of the polar plots can be arbitrarily chosen depending on the requirements or the desire of the user. The main limitation in this case is the value assigned to the frequency step $\Delta F$.

As long as a direct path between the two Vivaldi antennas is not interfered, this kind of measurement can be performed without taking into account additional parameters. In cases where the line of sight or when objects with conductive properties come closer to the antennas, it is possible to perceive relevant influences on the resulting radiation patterns.

\section{CONCLUSIONS AND DISCUSSION}

By using a simple combination of SFCW radar and a rotating stage attached to a stepper motor we have been able to obtain a 2D radiation pattern of a Vivaldi Antenna. This method has allowed the characterization of ultra-wide band Vivaldi antennas in the range from $1.0 \mathrm{GHz}$ to $6.0 \mathrm{GHz}$ by applying a modified version of the Friss-Equation. By using this combination we have been able to simplify the mathematical model for the antenna measurements.

Another advantage of this measuring method is that, if required, the radiation pattern of any given frequency within the characterization range can be chosen with the same resolution as the one configured on the SFCW radar.

With little or even no changes at all, this method can be applied for the characterization of different types of antennas as long as their working frequency resides within the bandwidth of the SFCW radar. 

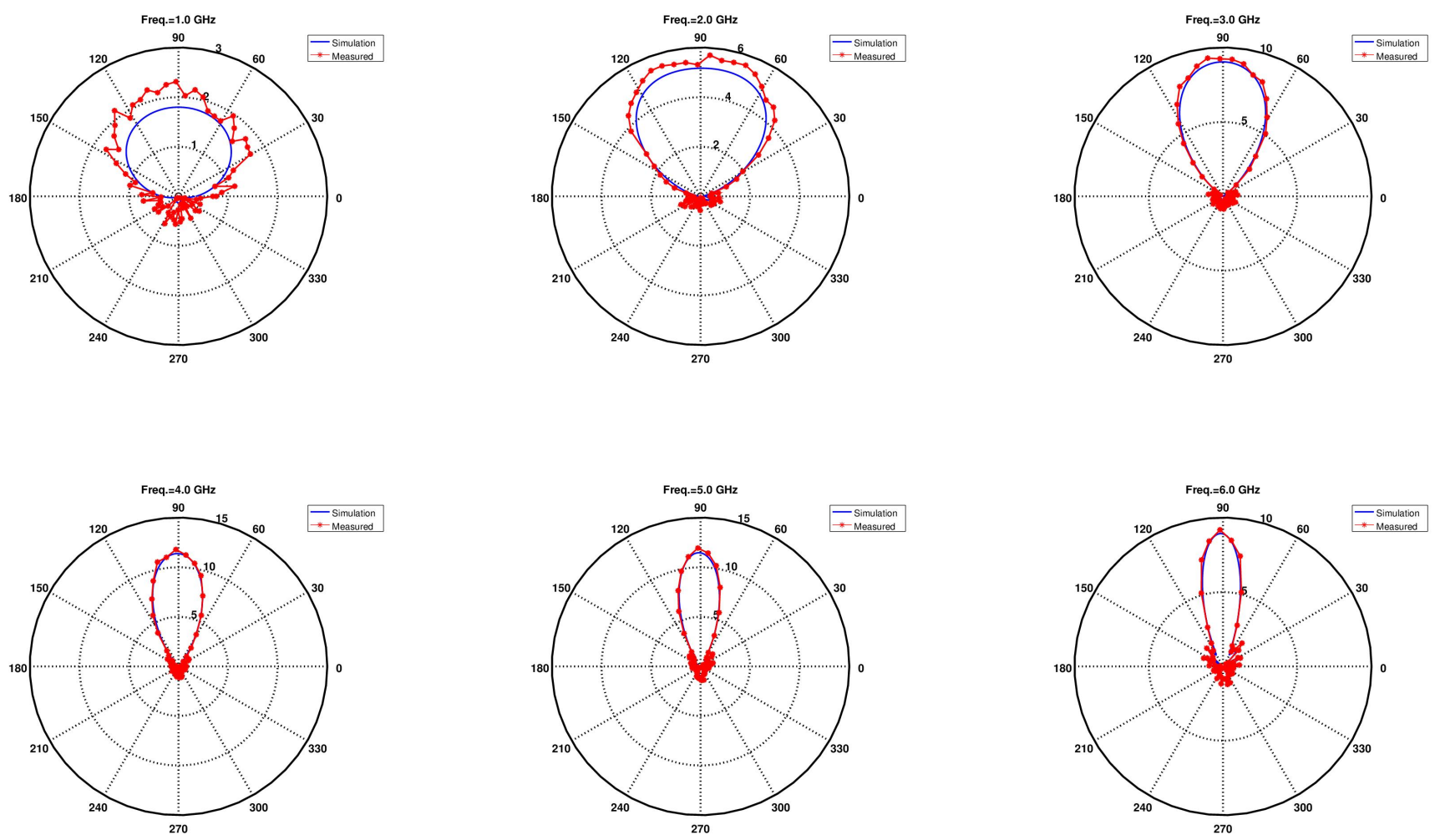

Fig. 4. Polar diagrams of the simulated and the measured radiation pattern of the Vivaldi antenna for the following frequencies: $1.0 \mathrm{GHz}, 2.0 \mathrm{GHz}, 3.0 \mathrm{GHz}$, 4.0 $\mathrm{GHz}, 5.0 \mathrm{GHz}$ and $6.0 \mathrm{GHz}$

These measurements have been easily achieved through the use of GNU/Octave scripts on a PC and they only take a few minutes (depending of the frequency steps and the angular resolution) and without using any special facilities such anechoic chambers or equipment (network or vector analyzers, power meters, HF sources, etc.), which reduces the time and costs related to these kind of characterizations.

This type of setup can be used as a fast, low-cost and simpler option for the characterization of antennas under the conditions aforementioned in this paper. Although the results presented in this document are limited to a $2 \mathrm{D}$ radiation pattern, with adequate mechanical apparatuses it can be extended for a full 3D measurement or the radiation pattern. As a consequence this will affect the measuring times and the amount of data gathered according to the amount of positions covered by the setup.

The setup might also have applications for determining the effects of different type of objects near the antennas. Objects that posses conductive properties generally modify the radiation patterns of the antennas. At simulations times, the effects of external objects are not always taken into account and therefore our method could help out in such a cases. However, this specific topic is rather material for further research.

\section{REFERENCES}

[1] A. M. J. Marindra, S. Promwong, and J. i. Takada, "Comprehensive characterization of a novel uwb elliptical planar monopole antenna," in TENCON 2012 IEEE Region 10 Conference, Nov 2012, pp. 1-6.

[2] B. Gabler, R. Horn, M. Jaeger, and A. Reigber, "Accurate antenna characterization for wideband synthetic aperture radar processing," in GeMiC 2014; German Microwave Conference, March 2014, pp. 1-4.

[3] C. Vasanelli, R. Batra, A. D. Serio, F. Boegelsack, and C. Waldschmidt, "Assessment of a millimeter-wave antenna system for mimo radar applications," IEEE Antennas and Wireless Propagation Letters, vol. 16, pp. 1261-1264, 2017.

[4] M. García-Fernández, Y. Álvarez López, A. Arboleya, B. González-Valdés, Y. Rodríguez-Vaqueiro, M. E. D. C. Gómez, and F. L.-H. Andrés, "Antenna diagnostics and characterization using unmanned aerial vehicles," IEEE Access, vol. 5, pp. 23 563-23 575, 2017.

[5] S. KUMAR and S. SHUKLA, WAVE PROPAGATION AND ANTENNA ENGINEERING:. Prentice Hall India Pvt., Limited, 2015.

[6] P. J. Gibson, "The vivaldi aerial," in 1979 9th European Microwave Conference, Sept 1979, pp. 101-105.

[7] H. Jol, Ground Penetrating Radar Theory and Applications. Elsevier Science, 2008.

[8] T. Denidni and G. Augustin, Ultrawideband Antennas for Microwave Imaging Systems:, ser. Artech House antennas and propagation library. Artech House, 2014.

[9] J. Eaton, D. Bateman, and S. Hauberg, The Gnu Octave 4.0 Reference Manual 1/2: Free Your Numbers. ARTPOWER International PUB, 2015. 